\title{
JAK3 Fusion Positive
}

National Cancer Institute

\section{Source}

National Cancer Institute. AKK3 Fusion Positive. NCI Thesaurus. Code C157589.

Indicates that expression of a fusion containing sequences from JAK3 has been detected in a sample. 